\title{
Jarilla-Coffea extract: a natural cosmetic product that improves eyelash and eyebrow growth in women
}

This article was published in the following Dove Medical Press journal:

Clinical, Cosmetic and Investigational Dermatology

\author{
María Rosario Alonso' \\ Silvia Pérez Damonte ${ }^{2}$ \\ Claudia Anesini' \\ 'Universidad de Buenos Aires, \\ Consejo Nacional de Investigaciones \\ Científicas y Técnicas (CONICET), \\ Instituto de Química y Metabolismo \\ del Fármaco (IQUIMEFA), Department \\ of Pharmacology, Facultad de Farmacia \\ y Bioquímica, Buenos Aires, Argentina \\ ${ }^{2}$ CLAIM, José Bonifacio 717 (CI424 \\ $\mathrm{CHO}$ ) Ciudad Autónoma de Buenos \\ Aires, Buenos Aires, Argentina
}

Correspondence: Claudia Anesini Universidad de Buenos Aires, Consejo Nacional de Investigaciones Científicas y Técnicas (CONICET), Instituto de Química y Metabolismo del Fármaco (IQUIMEFA), Departamento de Farmacología, Facultad de Farmacia y Bioquímica, Junín 956 2nd Floor, CP: III3, Buenos Aires, Argentina

$\mathrm{Tel}+5401149648347$

Fax +54 ol I 45083642

Email canesini@yahoo.com.ar
Purpose: A combination of extracts, from two plant species, ie, Coffea arabica and Larrea divaricata (Jarilla) (ECOHAIR ${ }^{\circledR}$ ), is being successfully used in Argentina as a cosmetic for hair recovery in androgenic and areata alopecia, and for eyelash and eyebrow growth. The objectives of this prospective study were to evaluate the capacity of Jarilla-Coffea extract gel of improving hair growth in relation to thickness, appearance of new hair, and hair length in comparison with a placebo in premenopausal and postmenopausal volunteers and to identify possible signs of ocular adverse local reactions related to the application of the gel.

Volunteers and methods: An open-label, placebo-controlled, prospective study was performed in healthy premenopausal and postmenopausal women during a daily administration period of 2 months (eyebrow growth) and 3 months (eyelash growth). The thickness of hair was determined using a video microscope MedicalScope ${ }^{\circledR}$. The appearance of new hairs and total area with hair in eyebrow and eyelash length were quantified using a photographic record with Fotofinder $^{\circledR}$ (Germany). The number of volunteers presenting variation in growth of new hair and length were also recorded.

Results: The product significantly increased the thickness of eyebrows ( $20 \%$ in $80 \%$ women) and eyelashes (19.44\% in $100 \%$ of women). The gel also increased the appearance of new hairs, total area with hair, and length but there was no statistical difference between treatment and placebo. Conclusion: The gel was capable of improving growth of eyelashes and eyebrows by inducing principally hair thickening without causing local adverse effects in a high percentage of volunteers.

Keywords: eyelash thickness, eyebrow thickness, plant extracts, menopause, adverse effects, cosmetic

\section{Introduction}

Both men and women suffer from hypotrichosis, which comprises either hair loss and/or thinning. This phenomenon, which affects not only the scalp but also eyelashes and eyebrows, is a result of an imbalance between cell proliferation and death. It is known that hair is involved in skin protection and thermal regulation. Especially, the eyelashes protect the eyes from light and dust, while the eyebrows protect the eyes from the sweat dripping down from the forehead. When hypotrichosis occurs, a deleterious effect on the patient's esthetic appearance also ensues. Hypotrichosis in eyelashes and eyebrows may be caused by ophthalmological infections and inflammatory process but also by dermatological diseases such as atopic dermatitis, contact dermatitis, psoriasis, lichen planus, and seborrheic dermatitis. Also hypotrichosis 
may be attributed to telogen effluvium caused by intake of drugs such as anticoagulants, lipid-lowering agents, antihypertensive agents (propranolol), valproate, barbiturates, bromocriptine, immunosuppressants, botulinum toxin, NSAIDs, and hormone imbalance. ${ }^{1}$

It is known that premenopausal, perimenopausal, and postmenopausal women suffer from hypotrichosis as a consequence of hormone misbalance; under this situation, some changes in skin such as increased dryness and roughness, decreased elasticity, warts, freckles, increased wrinkles, loss and graying of head hair, and lengthening of eyebrows and eyelashes are observed. ${ }^{2,3}$

Under these situations, extensions, masks of different types, eyelash bends, transplants, and permanent dyes are used on both eyelashes and eyebrows. One of the current products used to promote eyelash growth is a bimatoprost solution, which is a synthetic analog of prostaglandin F $2 \alpha$ used for the treatment of glaucoma. As side effect, bimatoprost is known to induce hair growth. The Food and Drug Administration (FDA) has approved it to be used in a $0.03 \%$ solution to stimulate the growth, thickness, and pigmentation of eyelashes in patients with hypotrichosis. However, the compound stimulates hair growth in some patients while in those with alopecia areata it is ineffective. On the other hand, when the product makes contact with the eye, serious adverse effects can occur. Analogs of prostaglandins (PGs) and prostanoids are associated with local and systemic effects not only when administered topically in the eye but also when placed in the eyelash insertion. These analogs can cause hyperpigmentation of the iris, as a consequence of an increase in melanogenesis, conjunctival hyperemia, eye dryness, erythema, itching, and uveitis. They have also been reported to cause headache, hirsutism, hepatic impairment, and to increase the sensitivity to herpes simplex virus (HSV) infection. In addition, it has been observed that both bimatoprost and latanoprost, other prostanoid, cause HSV dermatitis of the periocular skin. ${ }^{4}$ Taking these observations into account, the use of these prostanoids to promote the growth of the eyelashes is contraindicated in patients suffering from inflammatory diseases or from viral infections. Moreover, the commercially available products contain benzalkonium chloride as antibacterial, which has cytotoxic effect on the cornea and can cause erosion of the epithelium, irritation, and eye dryness. ${ }^{5}$

The prostanoids cannot be used during pregnancy because it has been classified by the FDA as a class $\mathrm{C}$ drug, since it is likely to cause malformations in the fetus. ${ }^{6}$

To date, no innocuous effective products to promote eyelash or eyebrow growth have been developed. A combination of extracts obtained from two plant species, ie, Coffea arabica and Larrea divaricata (Jarilla) (ECOHAIR ${ }^{\circledR}$ ), is being successfully used in Argentina, by Garre-Guevara Laboratory, as cosmetic, for hair recovery in scalp in cases of androgenic and areata alopecia and for eyelash and eyebrow growth. The effectiveness of the product in scalp alopecia has been verified not only in in vivo alopecia models in $\mathrm{C} 3 \mathrm{H}$ mice but also in a clinical trial. ${ }^{7,8}$

To provide scientific support for its effect in eyelash and eyebrow growth, the product was tested in premenopausal and postmenopausal healthy women. The primary objective of this prospective study was to evaluate the capacity of Jarilla-Coffea extract gel for improving hair growth in relation to thickness, appearance of new hairs, and hair length in comparison with a placebo. The secondary objective was to identify possible signs of ocular adverse local reactions related to the application of the gel.

\section{Volunteers and methods Study design}

An open-label, placebo-controlled, prospective study was performed to evaluate the efficacy of Jarilla-Coffea extract gel on eyelashes and eyebrows in premenopausal and postmenopausal healthy women during a daily administration period of 2 months, in the case of eyebrow growth, and 3 months, in the case of eyelash growth. These evaluation periods are in accordance with the product prospectus.

\section{Ethical considerations and good clinical practices}

This study was conducted in accordance with the intent and purpose of Good Clinical Practice regulations described in Title 21 of the US Code of Federal Regulations, the requirements of the Declaration of Helsinki (1964), Amendments Tokyo (1975), Venice (1983), and Hong Kong (1989), Seúl (2008), and for CLAIM Standard Operating Procedures and National Laws: "Guía de Buenas Prácticas de Investigación Clínica en Seres Humanos", in accordance to Resolution 1490/2007, published in "Boletín Oficial de la República Argentina" 14/11/2007, page 6 . The study was approved by a committee of independent ethics, which assessed the study protocol and informed consent. Once the protocol was approved by the independent Ethics Committee or institutional review board (CLAIM's Institutional Ethics Committee), the acceptance of the informed consent of the volunteer was obtained, in which, all the aspects making the study, all subjects and related research on which it requires information, and what was done with a terminology was explained in a clearly understandable manner to the volunteer. Informed consent form: Eyelashes and Eyebrows: Nr 0167-16. 


\section{Products under study}

The products used in this study were supplied by the GarreGuevara Laboratory. The products were identified as JarillaCoffea extract eyebrows (PE928061A) and Jarilla-Coffea extract eyelashes (PE038061) fashioner gels and placebo. Both gels were viscous lotions slightly colored with penetrating odor. The placebo used was glycerin USP.

\section{Volunteers}

Volunteers were recruited during a period of 3 months (July 2016 to September 2016). The study sample comprised ten women volunteers aged between 44 and 60 years (mean: $51.2 \pm 1.54)$. One volunteer was withdrawn from the study due to a lack of adherence, so the sample size was then reduced to nine volunteers.

Before enrolling in the trial, all volunteers were informed about the study and signed an informed consent. All volunteers were subjected to a thorough clinical revision. The following inclusion criteria were employed: 1) age: to be between 40 and 60 years; 2) volunteers with low density hair in eyelashes and eyebrows; 3 ) to have signed the informed consent form; and 4) volunteers who frequently used cosmetics and agreed to comply with the clinical trial requirements. Exclusion criteria were: 1) presence of any medical condition, or the ingestion or application of any medication by either the oral or the ocular route, such as steroidal and NSAIDs; 2 ) diagnosis of psoriasis, eczema, atopic dermatitis, folliculitis, lentigines, eye dryness, or ocular allergic diseases; 3 ) suffer from diabetes, asthma, or respiratory allergic diseases; 4) to have received treatments with immunosuppressant drugs as a consequence of organ transplantation; and 5) have a clinical record of cutaneous reactions after cosmetics application.

\section{Study objectives and assessments}

Volunteers were treated with Jarilla-Coffea extract eyebrow or eyelash gel on a daily basis, once a day, during 60 consecutive days with the eyebrow gel, or during 90 consecutive days with the eyelash gel. Two main variables were observed: 1) efficacy in relation to hair growth defined as thickness, appearance of new hair, area with hairs and hair length; and 2) safety and tolerability. The thickness of eyebrows and eyelashes was determined by a noninvasive methodology using a video microscope MedicalScope ${ }^{\circledR}$ with magnification of 200x. A calibrated micrometer scale having a least measurement of $0.01 \mathrm{~mm}$ was incorporated in the software of the video microscope and the diameter of at least ten hairs, from a same zone of the eyebrow or the eyelash, was measured always at the same position close to their bases using the measuring eyepiece. An average of the measurements was obtained for each volunteer. Images were registered under CD protocol no. 0164-16-12E1 (eyebrow) and no. 0164-1601E2 (for eyelash) on day $0\left(\mathrm{~T}_{0}\right)$ and on day $60\left(\mathrm{~T}_{60}\right)$ or on day $90\left(\mathrm{~T}_{90}\right)$, respectively. The appearance of new hairs and total area with hair, and eyelash length were quantified using a photographic record with Fotofinder ${ }^{\circledR}$ (Fotofinder Systems $\mathrm{GmbH}$, Bad Birnbach, Germany). The number of patients presenting variation in growth of new hair and length were also recorded.

Women were instructed to apply the product on the right eyebrows or eyelashes and to treat the left ones with the placebo, using the application instructions specified in the prospectus, that is: 1) daily application; 2) apply the product directly on the selective zone of eyebrows or eyelashes; 3 ) wash their hands between the application of placebo and treatment; 4) remove the excess of product; and 5) leave the product applied for 15-20 minutes. During the study period, women were forbidden to use other products or make up and to have sunbath.

\section{Safety studies}

Volunteers were subjected to ophthalmologic studies. First, a clinical history was obtained through examination of ocular surface with a slit lamp.

An evaluation of ocular surface was done after the treatment with the product. Daily appointments were made with each volunteer during the first and third week after treatment. The presence of two clinical signs and two symptoms were considered positive for side effects.

Scales of signs and symptoms that were used to evaluate side effects are shown in Table 1:

Table I

\begin{tabular}{|c|l|}
\hline $\begin{array}{l}\text { Scales of signs and symptoms } \\
\text { used to evaluate side effects }\end{array}$ & Description \\
\hline Symptoms score & \\
\hline 0 & Absent \\
\hline I & Present without discomfort \\
\hline 2 & $\begin{array}{l}\text { Present with discomfort without } \\
\text { interfering with daily activities }\end{array}$ \\
\hline 3 & $\begin{array}{l}\text { Present with discomfort and } \\
\text { interfering with daily activities }\end{array}$ \\
\hline Signs score & \\
\hline 0 & Absent \\
\hline I & Hardly visible \\
\hline 2 & Visible but not severe \\
\hline 3 & Visible and severe \\
\hline Local tolerance & \\
\hline 0 & Absent \\
\hline I & Hardly visible \\
\hline 2 & Visible but not severe \\
\hline 3 & Visible and severe \\
\hline
\end{tabular}


To determine the presence of signs and symptoms, an instrumental analysis was done with a tearscope, which allowed evaluating the lachrymal film stability through the interference of colors produced by the instrument on the lachrymal film when the film is disrupted. A crystallization test was also done, which allows determining the quality of the lachrymal film. Four types of lachrymal crystallization are known:

Type I: like a tree with ramifications.

Type II: like type I but with less ramifications with a few small leaves.

Type III: partial crystallization without fern formation.

Type IV: no crystallization.

\section{Statistical analysis}

A level of significance of 0.05 was employed in the hypothesis test. Percent variations (PVs) were determined for each volunteer employing the following formula:

Percent variation $=\left[\left(\right.\right.$ effect $T_{F} /$ effect $\left.\left.T_{0}\right)-1\right] \times 100$

The mean PV was calculated as the mean of individual $\mathrm{PV}(\mu \mathrm{PV}=[(\Sigma \mathrm{PV}) / \mathrm{n}]$, but not as PV of means PV $\mu=[(\mu$ $\left.\left.\mathrm{T}_{\mathrm{F}}\right) /\left(\mu \mathrm{T}_{0}\right)-1\right] \times 100$, where $\mathrm{T}_{0}$ : basal time, $\mathrm{T}_{\mathrm{F}}$ : time at 90 or 60 days of application. The mathematical analysis was done with IBM SPSS Statistics 22 statistical package.

To determine if the increase in the mean thickness during the treatment was greater than that obtained with the placebo, the linear generalized model for repeated measures (LGMRM) was applied. Results obtained with the product between $\mathrm{T}_{0}$ and $\mathrm{T}_{90}$ were analyzed with Student's $t$-test.

\section{Results}

\section{Effects on eyelashes}

\section{Eyelash thickness}

With the test product, the thickness means were 0.05 and 0.0594 at $\mathrm{T}_{0}$ and $\mathrm{T}_{90}$, respectively. This percent change was about 19.44\% (Table 2 and Figure 1A). All women displayed increased eyelash thickness.

For the placebo, the thickness mean for eyelashes was 0.05 at $\mathrm{T}_{0}$ and $\mathrm{T}_{90}$; the $\mathrm{PV}$ was $0 \%$ (Table 2 and Figure $1 \mathrm{~A}$ ). No women treated with placebo presented any change in eyelashes thickness. The results obtained with the active principle and the placebo are shown in a representative micrograph (Figure 2C).

To determine whether the increase in the mean thickness during the treatment was greater than that obtained with the placebo LGMRM was applied. The differences between
Table 2 Eyelash thickness: statistical descriptors

\begin{tabular}{|l|l|l|l|l|l|}
\hline & $\mathbf{N}$ & Minimum & Maximum & Mean & SD \\
\hline Active $\mathrm{T}_{0}$ & 9 & 0.04 & 0.06 & 0.0500 & 0.00707 \\
\hline Active $\mathrm{T}_{\mathrm{F}}$ & 9 & 0.05 & 0.07 & 0.0594 & 0.00635 \\
\hline $\mathrm{PV}_{\mathrm{F}} / \mathrm{T}_{0}$ & 9 & $8.33 \%$ & $25.00 \%$ & $19.44 \%$ & $4.93077 \%$ \\
\hline Placebo $\mathrm{T}_{0}$ & 9 & 0.04 & 0.07 & 0.0506 & 0.00950 \\
\hline Placebo $\mathrm{T}_{\mathrm{F}}$ & 9 & 0.04 & 0.07 & 0.0506 & 0.00950 \\
\hline $\mathrm{PVV}_{\mathrm{F}} / \mathrm{T}_{0}$ & 9 & $0.00 \%$ & $0.00 \%$ & $0.00 \%$ & $0.00000 \%$ \\
\hline
\end{tabular}

Abbreviation: $\mathrm{PV}$, percent variation.

treatment and placebo were highly significant $(P<0.001)$. Besides, the mean thickness values obtained with the product between $\mathrm{T}_{0}$ and $\mathrm{T}_{90}$ were significantly different $(P<0.001$, Student's $t$-test).

\section{Eyelash maximal length}

For the test product, the maximal eyelashes length means were $34.37 \mathrm{~mm}$ and $35.54 \mathrm{~mm}$ at $\mathrm{T}_{0}$ and $\mathrm{T}_{90}$, respectively. The percent change was $3.59 \%$ (Table 3 and Figure 1B). About $55.6 \%$ of volunteers presented an increase in the maximal eyelashes length.

For the placebo, the maximal eyelash length means were $36.09 \mathrm{~mm}$ and $35.54 \mathrm{~mm}$, at $\mathrm{T}_{0}$ and $\mathrm{T}_{90}$, respectively. The $\mathrm{PV}$ was $-1.09 \%$ (Table 3 and Figure 1B). The effects obtained with the active principle and the placebo are shown in a representative micrograph taken with a Fotofinder ${ }^{\circledR}$ camera (Figure 2A, B).

To determine whether the increase in the mean maximal length obtained after treatment with the product was higher than that obtained with the placebo LGMRM was applied. There was no difference between treatment and placebo $(P=0.11)$.

\section{Effects on eyebrows Eyebrow thickness}

The active principle induced an increase in the eyebrow thickness of $20 \%$ in $80 \%$ of volunteers (Table 4 and Figure $3 \mathrm{~A}, \mathrm{~B}, \mathrm{E}, \mathrm{F})$. The eyebrow thickness means were 0.055 and 0.06 at $\mathrm{T}_{0}$ and $\mathrm{T}_{60}$, respectively. A PV mean of $9.16 \%$ was observed (Table 4).

For the placebo, the mean thickness was 0.058 at $\mathrm{T}_{0}$ and $\mathrm{T}_{60}$. No PV was assigned in $100 \%$ of the volunteers (Table 4 and Figure 3A, B, E, F).

To determine whether the increase in the mean thickness after the treatment was higher than that obtained with the placebo LGMRM was applied. There was difference between mean values after treatment with the product and the placebo $(P<0.001)$. 

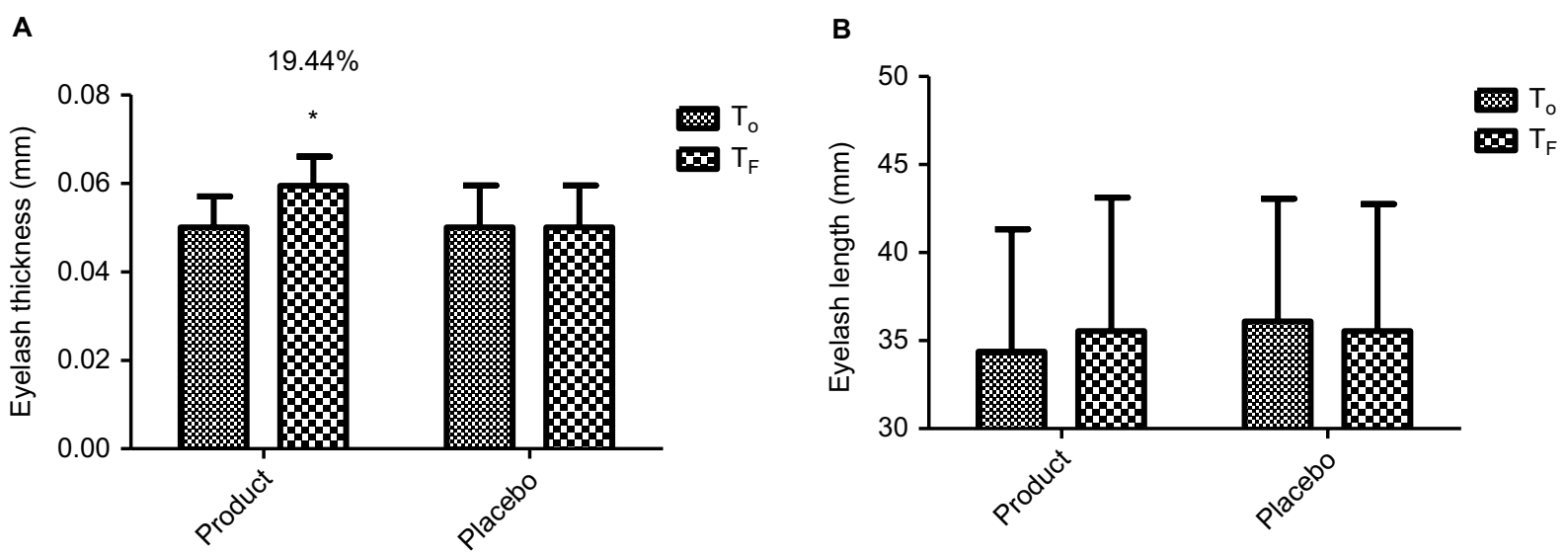

Figure I Effect of eyelash gel on (A) eyelash thickness and (B) length.

Notes: The product effect was compared with a placebo. Results represent the mean $\pm S D$ of nine determinations. $* P<0.05$ : significant differences between $T_{0}$ and $T_{90}$ (Student's t-test)

Table 3 Eyelash maximal length: statistical descriptors

\begin{tabular}{|l|l|l|l|l|l|}
\hline $\begin{array}{l}\text { Statistical } \\
\text { descriptors }\end{array}$ & $\mathbf{N}$ & Minimum & Maximum & Mean & SD \\
\hline Active $\mathrm{T}_{0}$ & 9 & 21.5720 & 44.3710 & 34.3730 & 6.9594393 \\
\hline Active $\mathrm{T}_{\mathrm{F}}$ & 9 & 22.7930 & 46.4040 & 35.5494 & 7.5886040 \\
\hline $\mathrm{PV}_{\mathrm{F}} / \mathrm{T}_{0}$ & 9 & -5.13 & 23.92 & $3.59 \%$ & 9.04619 \\
\hline Placebo $\mathrm{T}_{0}$ & 9 & 22.7930 & 43.9620 & 36.0910 & 6.9738635 \\
\hline${\text { Placebo } \mathrm{T}_{\mathrm{F}}} 9$ & 24.8320 & 47.2160 & 35.5494 & 7.2176550 \\
\hline $\mathrm{PV}_{\mathrm{F}} / \mathrm{T}_{0}$ & 9 & -13.67 & 8.95 & $-1.09 \%$ & 8.65175 \\
\hline
\end{tabular}

Abbreviation: $\mathrm{PV}$, percent variation.

\section{Eyebrow new hairs}

A $60 \%$ of women treated with the gel presented an increase in the number of hairs in the eyebrows; the mean new hair values were 119 and 130 at $\mathrm{T}_{0}$ and $\mathrm{T}_{60}$, respectively, with a mean increment value of $12.57 \%$ and a maximal increment of 56\% (Table 5 and Figure 3A-D). Moreover, the mean values of new hairs obtained with the placebo were 118 and 123 , at $\mathrm{T}_{0}$ and $\mathrm{T}_{60}$, respectively, with a percent increase of $3.94 \%$ and a maximal increment of $62 \%$. In this case, $50 \%$ of volunteers presented a change.

To determine whether the increase in the mean value of new hairs after the treatment was higher than that obtained with the placebo LGMRM was applied. There was no difference between treatment and placebo $(P=0.749)$.

\section{Total area with hair in eyebrows}

The mean total areas with hair obtained after treatment with the product were 0.435 and 0.444 , for $\mathrm{T}_{0}$ and $\mathrm{T}_{60}$, respectively. The mean percent increase was $15.53 \%$, with a maximum value of $132 \%$ (Table 6 ). This change was observed in $50 \%$ of volunteers (Figure 3C, D).

For the placebo, the total areas with hair means were 0.400 and 0.418 for $\mathrm{T}_{0}$ and $\mathrm{T}_{60}$, respectively. The mean percent increase was $5.69 \%$, with a maximum value of $84 \%$. This change was observed in $50 \%$ of volunteers (Figure 3C, D).

To determine whether the increase in the mean total area with hair after the treatment with the product was greater than that obtained with the placebo LGMRM was applied. There was no difference between the total area with hair between treatment and placebo $(P=0.907)$.

\section{Safety studies}

When applied on the eyelashes, the product did not cause any ocular adverse reactions. The lachrymal film in all volunteers did not present any alteration. The crystallization was type I in all cases. In addition, there was no difference - in the effects obtained on the eyelids and the orbital ledge skin - of product treated and placebo.

\section{Discussion}

In this work, the efficacy of Jarilla-Coffea extract eyelashes and eyebrow gels was demonstrated. In all healthy premenopausal and postmenopausal women with scant eyelashes and eyebrows, both these gels were capable of increasing the eyelash and eyebrow growth in relation to thickness in all women treated, improving the hair appearance by this way. Moreover, other parameters such as the eyelash maximal length (that increased in $55.6 \%$ of the volunteers) and the eyebrow new hairs (in $60 \%$ of the volunteers) related to growth were modified by this gel. However, there was no statistical difference with the placebo ( $P=0.11$ and 0.749 , respectively).

It could be possible that the product had increased thickness of eyelash and eyebrow hair by promoting hair from telogen to anagen phase. Previously, it was demonstrated that the plant extracts, present in the gel, were capable to induce hair 
A

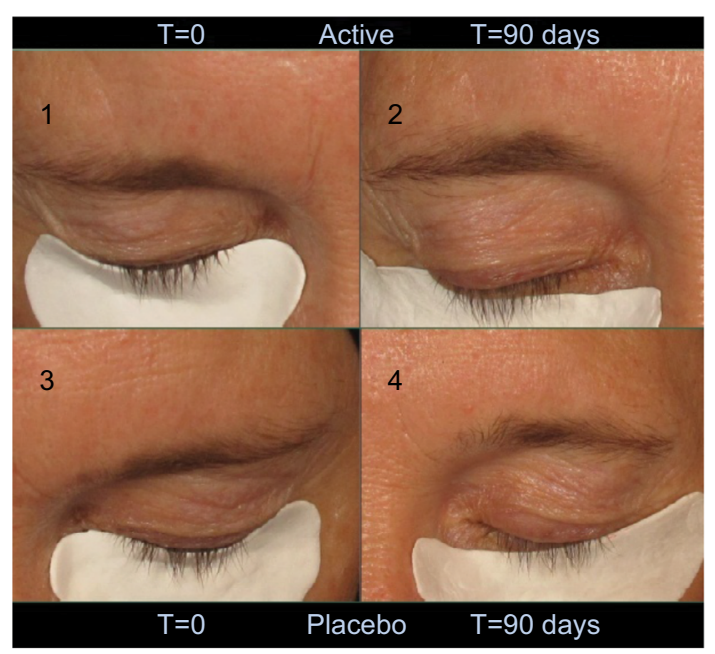

B

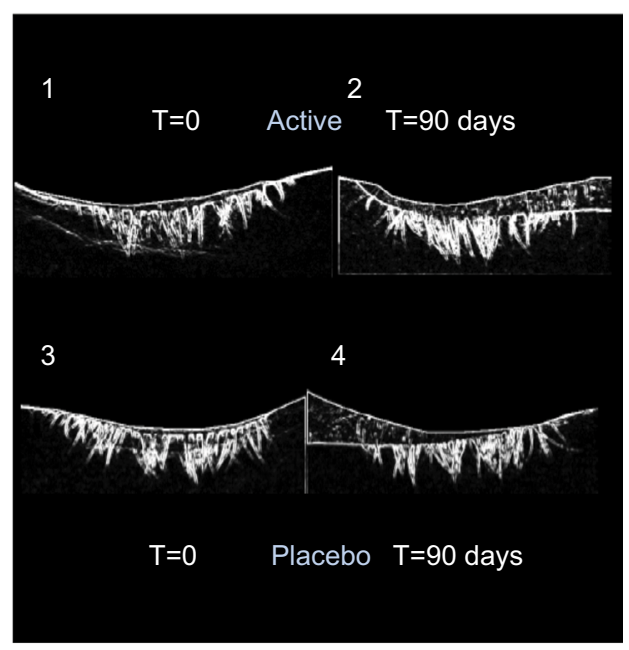

C

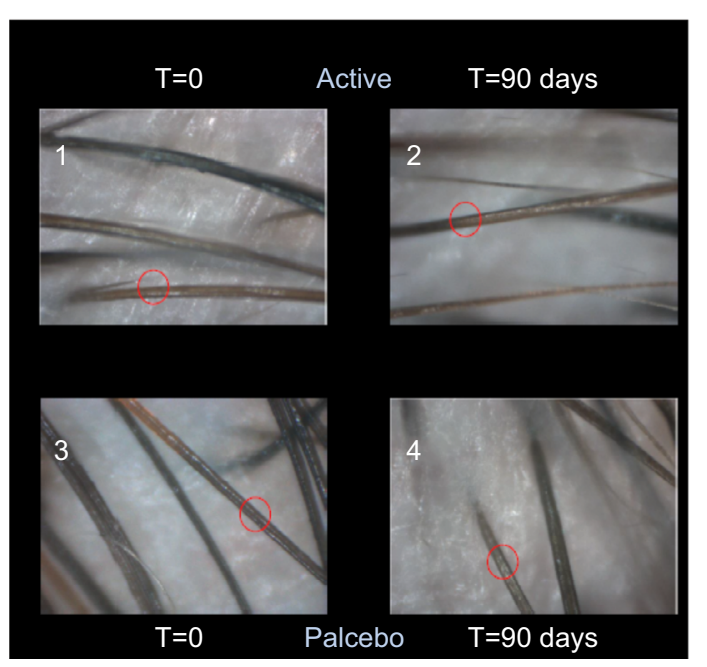

Figure 2 Effect of eyelash gel on (A, B) eyelash length and (C) thickness.

Notes: (A) Representative micrographs of eyelashes treated either with gel (upper) or with placebo (lower). Micrographs were taken with Fotofinder ${ }^{\circledR}$. (B) Analysis of the micrographs shown in (A). (C) Representative micrographs of eyelashes treated with either gel (upper) or with placebo (lower). Analysis of thickness was done with video microscope. I: $\mathrm{T}_{0} ; 2: \mathrm{T}_{90}$ treated with gel; $3: \mathrm{T}_{0} ; 4: \mathrm{T}_{90}$ treated with placebo. $\mathrm{T}_{0}$ : initial time, $\mathrm{T}_{90}$ : final time.

Table 4 Eyebrow thickness: statistical descriptors

\begin{tabular}{|l|l|l|l|l|l|}
\hline $\begin{array}{l}\text { Statistical } \\
\text { descriptors }\end{array}$ & $\mathbf{N}$ & Minimum & Maximum & Mean & SD \\
\hline Active $\mathrm{T}_{0}$ & 10 & 0.04 & 0.07 & 0.0550 & 0.00850 \\
\hline Active $\mathrm{T}_{60}$ & 10 & 0.04 & 0.07 & 0.0600 & 0.009129 \\
\hline Active $\mathrm{PV}$ & 10 & $0.00 \%$ & $20.00 \%$ & $9.1667 \%$ & $6.19886 \%$ \\
\hline Placebo $\mathrm{T}_{0}$ & 10 & 0.04 & 0.07 & 0.0580 & 0.01033 \\
\hline Placebo $\mathrm{T}_{60}$ & 10 & 0.04 & 0.07 & 0.0580 & 0.01033 \\
\hline Placebo PV & 10 & $0.00 \%$ & $0.00 \%$ & $0.0000 \%$ & $0.00000 \%$ \\
\hline
\end{tabular}

Abbreviation: PV, percent variation.

from telogen to anagen phase in $\mathrm{C} 3 \mathrm{H}$ mice. ${ }^{7}$ Moreover, the capacity of a lotion, made with the two extracts, in stimulating hair growth in humans has previously been determined. Such study was conducted in patients with noncicatricial alopecia in a randomized, prospective, open-label, single-branch, phase IV cohort study. The clinical study revealed that the lotion improves the appearance, volume and appearance of new hair due to an induction of hair growth, and a decrease of hair loss (from the moment of its application) in $84.6 \%$ of patients (total patients 52), after 90 days of treatment. The decrease in hair loss was higher in women, and the best results were obtained in grades I and II androgenic alopecia and in alopecia areata. ${ }^{8}$

The mechanism of the extracts involved in promoting anagen phase could be related to a modulation of PGs. It was demonstrated that unlike PGD2, which has an inhibitory effect, prostaglandin F2 $\alpha$ and PGF2 induce hair growth. Therefore, PGs may be considered a pharmacological target for the treat- 

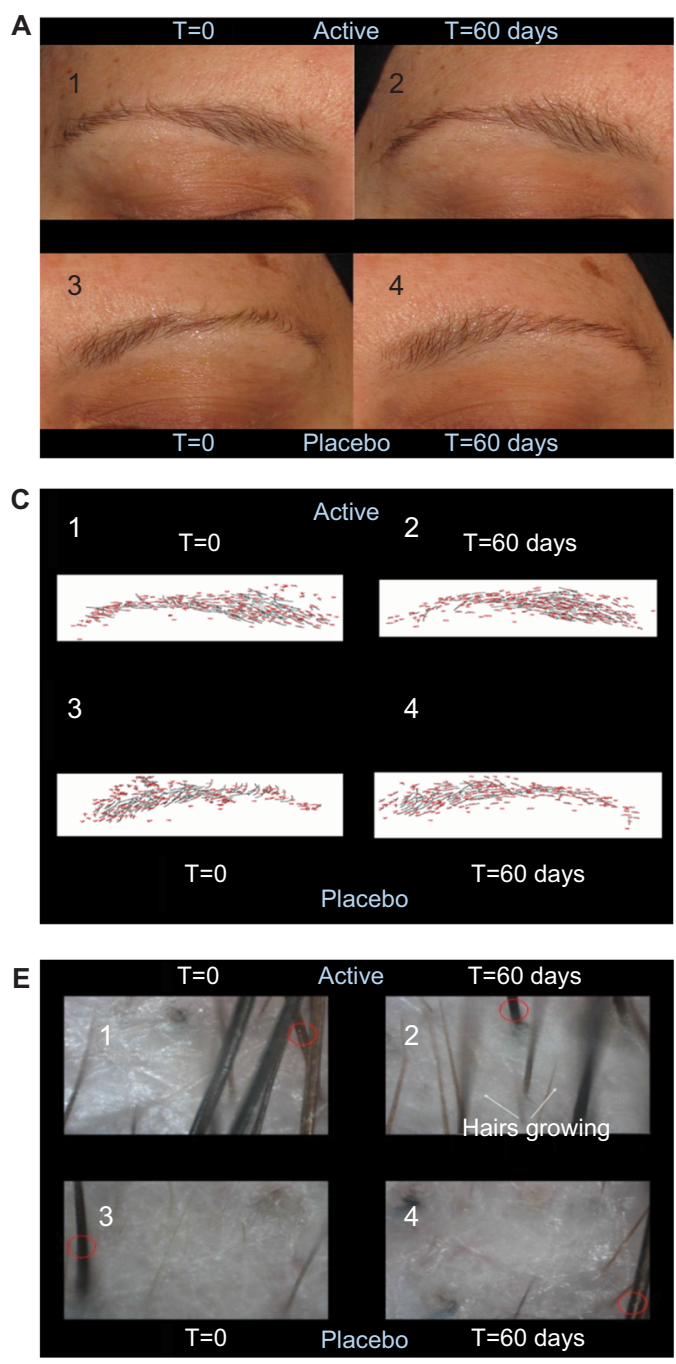

B

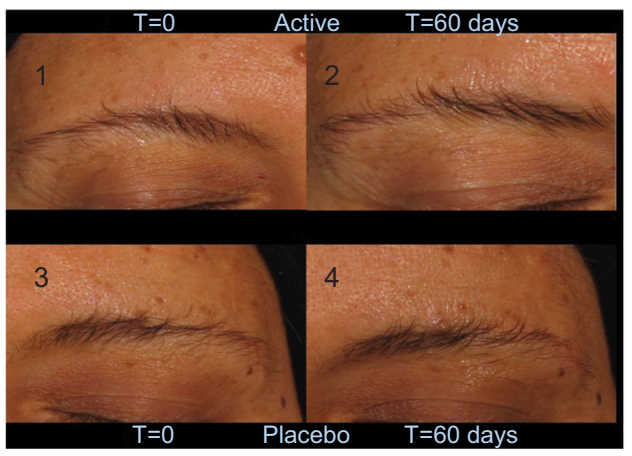

D

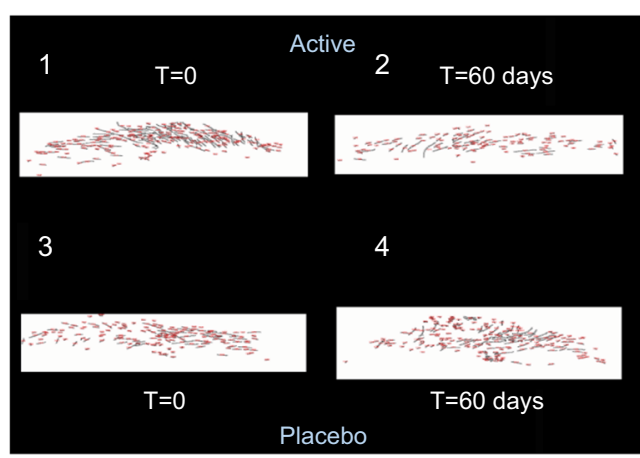

F

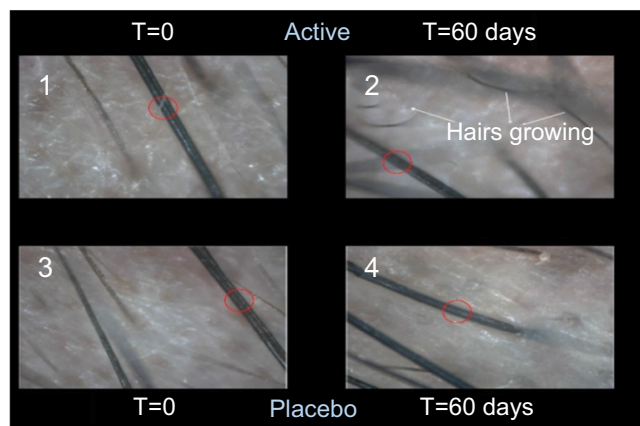

Figure 3 Effect of eyebrow gel on (A-D) eyebrow growth and (E, F) thickness.

Notes: (A, B) Representative micrographs of eyebrows treated either with gel (upper) or with placebo (lower). Micrographs were taken with Fotofinder ${ }^{\circledR}$. (C, D) Analysis of the micrographs shown in (A, B). (E, F) Representative micrographs of eyebrows treated either with gel (upper) or with placebo (lower). Analysis of thickness was done with video microscope. I: $\mathrm{T}_{0} ; 2: \mathrm{T}_{60}$ treated with gel; 3: $\mathrm{T}_{0} ; 4: \mathrm{T}_{60}$ treated with placebo. $\mathrm{T}_{0}$ : initial time, $\mathrm{T}_{60}$ : final time.

Table 5 Eyebrow new hairs: statistical descriptors

\begin{tabular}{|l|l|l|l|l|l|}
\hline $\begin{array}{l}\text { Statistical } \\
\text { descriptors }\end{array}$ & $\mathbf{N}$ & Minimum & Maximum & Mean & SD \\
\hline Active $\mathrm{T}_{0}$ & 10 & 56 & 204 & 119.70 & 57.337 \\
\hline Active $\mathrm{T}_{F}$ & 10 & 60 & 269 & 130.70 & 66.369 \\
\hline Active $\mathrm{PV}$ & 10 & $-36 \%$ & $56 \%$ & $12.57 \%$ & $29.776 \%$ \\
\hline Placebo $\mathrm{T}_{0}$ & 10 & $6 \mathrm{I}$ & 162 & $\mathrm{I} 8.30$ & 32.308 \\
\hline Placebo $\mathrm{T}_{\mathrm{F}}$ & 10 & 53 & 206 & 123.80 & $49.57 \mathrm{I}$ \\
\hline Placebo PV & 10 & $-24 \%$ & $62 \%$ & $3.94 \%$ & $27.362 \%$ \\
\hline
\end{tabular}

Abbreviation: $\mathrm{PV}$, percent variation.

ment of androgenetic alopecia. ${ }^{9,10}$ In line with these findings, it was found that inhibitors of PGs synthesis, such as aspirin, indomethacin, and ibuprofen, can inhibit hair growth. ${ }^{11,12}$ The extracts present in the gel have flavonoids (quercetin derivatives)
Table 6 Eyebrow total area with hair: statistical descriptors

\begin{tabular}{|l|l|l|l|l|l|}
\hline $\begin{array}{l}\text { Statistical } \\
\text { descriptors }\end{array}$ & $\mathbf{N}$ & Minimum & Maximum & Mean & SD \\
\hline Active $\mathrm{T}_{0}$ & 10 & 0.15924 & 0.84720 & 0.4350210 & 0.23240888 \\
\hline Active $\mathrm{T}_{\mathrm{F}}$ & 10 & 0.17062 & 0.76764 & 0.4448500 & 0.20246945 \\
\hline Active $\mathrm{PV}$ & 10 & $-51.13 \%$ & $132.60 \%$ & $15.5322 \%$ & $51.52571 \%$ \\
\hline Placebo $\mathrm{T}_{0}$ & 10 & 0.14609 & 0.75613 & 0.4002340 & 0.16880262 \\
\hline Placebo $\mathrm{T}_{\mathrm{F}}$ & 10 & 0.12053 & 0.75707 & 0.4184350 & 0.19337064 \\
\hline Placebo PV & 10 & $-24.83 \%$ & $84.87 \%$ & $5.6920 \%$ & $33.34614 \%$ \\
\hline
\end{tabular}

Abbreviation: $\mathrm{PV}$, percent variation.

and nordihydroguaiaretic acid (NDGA) - compounds that can modulate endogen PGs. For example, NDGA is an inhibitor of lipooxigense, therefore, this polyphenol could increase the PG synthesis, by activating cyclooxigenase (COX) pathway, thus 
increasing eyelash growth. Moreover, and depending on its concentration, NDGA may also inhibit the synthesis of PGD2 through the inhibition of COX-1. In addition, it has also been demonstrated that the flavonoids present in Chinese herbs, such as amentoflavone, quercetin-3-O-rutinoside (rutin), and hinokiflavone, are PGD2 inhibitors with good pharmacokinetic properties and high efficacy in the treatment of hair loss. ${ }^{13}$

By other way, it is known that ROS can modulate the balance between the anagen and telogen phases, causing damage to DNA, cell proteins, and lipids leading to cell arrest and follicular cell death. ${ }^{14}$ These events are known to promote the hair follicle to shift from the anagen to the telogen phase, or hair loss phase. Normally, the ROS levels are controlled by the activity of antioxidant enzymes, such as superoxide dismutase (SOD), catalase (CAT), and peroxidase (Px); however, there are cases in which the ROS production exceeds their metabolism rate leading to different diseases. It is known that the superoxide anion is involved in the occurrence of alopecia, since this free radical interferes with the production of nitric oxide, which is an essential factor for hair growth. ${ }^{15}$

The extracts present in the gel are also known to have antioxidant activities, such as Px, CAT, and SOD-like activities. ${ }^{16,17}$ Therefore, the effects observed after the treatment with the gel could also be related to these biological activities exerted by the extracts.

It is thought that herb-based formulations may influence hair growth by stimulating or inhibiting a variety of growth factors, cytokines, hormones, enzymes, as well as through the modulation of signaling pathways. Some herbs and their active constituents allow maintaining the hair growth cycle in the anagen phase, while others inhibit hair apoptosis during the catagen phase.

The fact that the product increased hair length and the appearance of new hairs but not in a significant manner suggests that a longer treatment is necessary.

It is noteworthy that neither ocular nor dermal side effects were observed with Jarilla-Coffea extract gel in contrast with the products used nowadays to increase eyelash or eyebrow growth or for alopecia treatment.

\section{Conclusion}

This study concludes that the Jarilla-Coffea extract gel was capable of improving eyelash and eyebrow growth by inducing hair thickness, and also the appearance of new hairs and hair length were changed, without exerting local adverse effects.

\section{Acknowledgment}

The authors thank Laboratory Garre-Guevara SRL Argentina for the financial support of the study.

\section{Author contributions}

CA was involved in conception of the study and performed analysis and interpretation of data. Moreover, he contributed toward data analysis, drafting, and critically revising the manuscript and agrees to be accountable for all aspects of the work. MRA was involved in extract preparation and quality control of products and analysis and interpretation of data. SPD planned the trial performing visits and instrumental evaluation and protocol design. All authors contributed to data analysis, drafting or revising the article, gave final approval of the version to be published, and agree to be accountable for all aspects of the work.

\section{Disclosure}

The authors report no conflicts of interest in this work.

\section{References}

1. Vij A, Bergfeld WF. Madarosis, milphosis, eyelash trichomegaly, and dermatochalasis. Clin Dermatol. 2015;33(2):217-226.

2. Naito A, Sato T, Matsumoto T, et al. Dihydrotestosterone inhibits murine hair growth via the androgen receptor. $\mathrm{Br} J$ Dermatol. 2008;159(2):300-305.

3. Marks R, Shahrad P. Skin changes at the time of the climacteric. Clin Obstet Gynecol. 1977;4:207-226.

4. Wand M. Latanoprost and hyperpigmentation of eyelashes. Arch Ophthalmol. 1997;115(9):1206-1208.

5. Baudouin C, Riancho L, Warnet JM, Brignole F. In vitro studies of antiglaucomatous prostaglandin analogues: travoprost with and without benzalkonium chloride and preserved latanoprost. Invest Opthalmol Vis Sci. 2007;48(9):4123-4128.

6. Xue-Gang XU, Chen HD. Prostanoids and hair follicles: implications for therapy of hair disorders. Acta Derm Venereol. 2018;98(3):318-323.

7. Davicino R, Alonso MR, Anesini C. Composiciones tópicas para el crecimiento del cabello [Topical compositions for growth hair related]. Patente 090101704. Boletín de Patentes. 2010;44.

8. Alonso MR, Anesini C. Clinical evidence of increase in hair growth and decrease in hair loss without adverse reactions promoted by the commercial lotion ECOHAIR®. Skin Pharmacol Physiol. 2017;30(1):46-54.

9. Deplewski D, Rosenfield RL. Role of hormones in pilosebaceous unit development. Endocr Rev. 2000;21(4):363-392.

10. Colombe L, Vindrios A, Michelet JF, Bernard BA. Prostaglandin metabolism in human hair follicle. Exp Dermatol. 2007;16(9):762-769.

11. Meyer HC. Alopecia associated with ibuprofen. JAMA. 1979;242(2):142.

12. Pillans PI, Woods DJ. Drug-associated alopecia. Int J Dermatol. 1995;34:149-158.

13. Fong P, Tong HH, Ng KH, Lao CK, Chong CI, Chao CM. In silico prediction of prostaglandin D2 synthase inhibitors from herbal constituents for the treatment of hair loss. J Ethnopharmacol. 2015; 175:470-480.

14. Hogg N. Free radicals in disease. Semin Reprod Med. 1998;16(4):241-248.

15. Liu N, Wang LH, Guo LL, et al. Chronic restraint stress inhibits hair growth via substance $\mathrm{P}$ mediated by reactive oxygen species in mice. PLoS One. 2013;8(4):e61574.

16. Anesini C, Turner S, Borda E, Ferraro G, Coussio J. Effect of Larrea divaricata Cav. extract and nordihydroguaiaretic acid upon peroxidase secretion in rat submandibulary glands. Pharmacol Res. 2004;49(5):441-448.

17. Ramalakshmi K, Rahath Kubra I, Jagan Mohan Rao L. Antioxidant potential of low-grade coffee beans. Food Res Int. 2008;41(1):96-103. 


\section{Publish your work in this journal}

Clinical, Cosmetic and Investigational Dermatology is an international, peer-reviewed, open access, online journal that focuses on the latest clinical and experimental research in all aspects of skin disease and cosmetic interventions. This journal is included on PubMed. The manuscript management system is completely online and includes a very quick and fair peer-review system, which is all easy to use. Visit http://www.dovepress.com/testimonials.php to read real quotes from published authors

Submit your manuscript here: https://www.dovepress.com/clinical-cosmetic-and-investigational-dermatology-journal 